\title{
Existence of Homoclinic Orbits for a Singular Differential Equation Involving $p$-Laplacian
}

\author{
Honghui Yin $\mathbb{D}^{1},{ }^{1}$ Bo Du $\left(\mathbb{D},{ }^{1}\right.$ Qing Yang, ${ }^{1}$ and Feng Duan ${ }^{2}$ \\ ${ }^{1}$ School of Mathematics and Statistics, Huaiyin Normal University, Huaian Jiangsu 223300, China \\ ${ }^{2}$ Basic Education Department, Tongling Polytechnic, Tongling Anhui 244061, China \\ Correspondence should be addressed to Bo Du; dubo7307@163.com
}

Received 10 April 2020; Accepted 26 June 2020; Published 20 July 2020

Academic Editor: Gisele Mophou

Copyright (c) 2020 Honghui Yin et al. This is an open access article distributed under the Creative Commons Attribution License, which permits unrestricted use, distribution, and reproduction in any medium, provided the original work is properly cited.

\begin{abstract}
The efficient conditions guaranteeing the existence of homoclinic solutions to second-order singular differential equation with $p$-Laplacian $\left(\phi_{p}\left(x^{\prime}(t)\right)\right)^{\prime}+f\left(x^{\prime}(t)\right)+g(x(t))+(h(t) / 1-x(t))=e(t)$ are established in the paper. Here, $\phi_{p}(s)=|s|^{p-2} s$, $p>1, f, g, h, e \in C(\mathbb{R}, \mathbb{R})$ with $h(t+T)=h(t)$. The approach is based on the continuation theorem for coincidence degree theory.
\end{abstract}

\section{Introduction}

In the last years, homoclinic solutions for Hamiltonian systems and differential and difference systems have been studied by several authors. Based on variational methods and critical points theory, Rabinowitz [1] has given fundamental contributions to homoclinic solutions for Hamiltonian systems. Carriãro and Miyagaki [2] obtained the existence of homoclinic orbits for second-order timedependent Hamiltonian systems. Izydorek and Janczewska [3] obtained that a homoclinic orbit is obtained as a limit of $2 k T$-periodic solutions of a certain sequence of the second-order differential equations. By means of an extension of Mawhin's continuation theorem, Lu et al. [4] obtained the existence of homoclinic solutions for a class of second-order neutral functional differential systems. Ding and Guo [5] showed that there exists at least one homoclinic solution for the anomalous diffusion system. For more results about homoclinic solutions, see, e.g., [6-10] and relevant references.

In recent years, homoclinic solution problems of secondorder singular differential equation have raised concerns. Bonheure and Torres [11] studied the existence of homocli- nic solutions for the model scalar second-order boundary value problem

$$
-u^{\prime \prime}+c(x) u^{\prime}+a(x) u=\frac{b(x)}{u^{P}(x)}
$$

where $a, b, c \in C(\mathbb{R}, \mathbb{R}), p>0$. When $c=0$, Equation (1) has a good variational structure and can be studied by variational method for Equation (1), see $[1,12,13]$. When $c \neq 0$, variational method cannot be used to study Equation (1) because of the no good variational structure. Hence, based on the method of the upper and lower solutions and fixed point theorem on cones, the authors obtained the existence of homoclinic solutions for Equation (1) which is different from the variational methods used in [14-16].

Motivated by the above work, this paper is devoted to the study of the existence of homoclinic solutions to secondorder singular differential equation with $p$-Laplacian:

$$
\left(\phi_{p}\left(x^{\prime}(t)\right)\right)^{\prime}+f\left(x^{\prime}(t)\right)+g(x(t))+\frac{h(t)}{1-x(t)}=e(t),
$$


where $\phi_{p}(s)=|s|^{p-2} s, p>1, f, g, h, e \in C(\mathbb{R}, \mathbb{R})$ with $h(t+T)$ $=h(t)>0$. As in the literature, a solution $u(t)$ of Equation (2) is called a homoclinic solution if $u(t) \longrightarrow 0$ as $|t| \longrightarrow$ $+\infty$. When such a solution satisfies in addition to $u^{\prime}(t)$ $\longrightarrow 0$ as $|t| \longrightarrow+\infty$, it is usually called a homoclinic solution or a pulse, although here, 0 is not a stationary solution of Equation (2). Since Equation. (2) is a strongly nonlinear equation, the traditional methods (including fixed point theorem and lower and upper solutions) are no longer applicable to study homoclinic solutions to Equation (2), so a new continuation theorem due to Mana'sevich and Mawhin will be developed for studying Equation (2).

The distinctive contributions of this paper are outlined as follows:

(1) The problem (2) is a more general form compared with existing problems (see $[1,11-13])$. Hence, the results of this paper can be extended to other more specific problems

(2) Due to singularity, it is very difficult for estimating priori bound. In order to overcome this difficulty, we develop a new technique introduced in [17] for continuation theorem

(3) A unified framework is established to handle second-order equations with singularity term and $p$-Laplacian operator

The following sections are organized as follows: In Section 2, some useful lemmas and notations are given. In Section 3, sufficient conditions are established for the existence of homoclinic solutions of (2). In Section 4, two examples are given to show the feasibility of our results. Finally, Section 5 concludes the paper.

\section{Preliminary and Some Lemmas}

In this section, we give some notations and lemmas which will be used in this paper. The set of all positive integers is denoted by N. Let

$$
C_{T}=\{x \mid x \in C(\mathbb{R}, \mathbb{R}), x(t+T) \equiv x(t), \forall t \in \mathbb{R}\}
$$

with the norm $|\varphi|_{0}=\max _{t \in[0, T]}|\varphi(t)|, \forall \varphi \in C_{T}$. When $p \neq 2$, $p$ Laplacian $\left(\phi_{p}\left(s^{\prime}\right)\right)^{\prime}$ in $(2)$ is a nonlinear operator, the famous Mawlin's continuation theorem [18] cannot be directly applied to (2). In order to generalize Mawlin's continuation theorem, Mana'sevich and Mawhin [17] obtained the following continuation theorem for nonlinear systems with $p$-Laplacian-like operators:

(1) For each $\lambda \in(0,1)$, the problem

$$
\left(\phi_{p}\left(u^{\prime}\right)\right)=\lambda f\left(t, u, u^{\prime}\right), u(0)=u(T), u^{\prime}(0)=u^{\prime}(T),
$$

has no solution on $\partial \Omega$
(2) The equation

$$
\mathscr{F}(a)=\frac{1}{T} \int_{0}^{T} f(t, a, 0) d t=0,
$$

has no solution on $\partial \Omega \cap \mathbb{R}^{N}$

(3) The Brouwer degree

$$
d_{B}\left(\mathscr{F}, \Omega \cap \mathbb{R}^{N}, 0\right) \neq 0
$$

Theorem 1. Assume that $\Omega$ is an open bounded set in $C_{T}$ such that the above conditions (4) - (6) hold.

Then, problem

$$
\left(\phi_{p}\left(u^{\prime}\right)\right)=f\left(t, u, u^{\prime}\right), u(0)=u(T), u^{\prime}(0)=u^{\prime}(T),
$$

has a solution in $\bar{\Omega}$.

Lemma 2 (see [19]). If $u \in C(\mathbb{R}, \mathbb{R}), a>0, \mu_{1}, \mu_{2}>1$ are constants, then for every $t \in \mathbb{R}$, the following inequality holds:

$$
\begin{aligned}
u(t) \leq & (2 a)^{-1 / \mu_{1}}\left(\int_{t-a}^{t+a}|u(s)|^{\mu_{1}} d s\right)^{1 / \mu_{1}} \\
& +a(2 a)^{-1 / \mu_{2}}\left(\int_{t-a}^{t+a}\left|u^{\prime}(s)\right|^{\mu_{2}} d s\right)^{1 / \mu_{2}} .
\end{aligned}
$$

Lemma 3 (see [4]). Let $\left\{u_{k}\right\} \in C_{2 k T}^{1}$ be a sequence of $2 k T$ periodic functions, such that for each $k \in N, u_{k}$ satisfies

$$
\left|u_{k}\right|_{0} \leq A_{0},\left|u_{k}{ }^{\prime}\right|_{0} \leq A_{1}
$$

where $A_{0}, A_{1}$ are constants independent of $k \in N$. Then, there exist $u_{0} \in C(\mathbb{R}, \mathbb{R})$ and a subsequence $\left\{u_{k_{j}}\right\}$ of $\left\{u_{k}\right\}$ such that for each $j \in N$

$$
\max _{t \in[-j T, j T]}\left|u_{k_{i}}(t)-u_{0}(t)\right| \longrightarrow 0 \text { as } i \longrightarrow+\infty
$$

For investigating the existence of homoclinic solutions to (2), for each $k \in N$, we firstly consider the existence of $2 k T$-periodic solutions $u_{k}(t)$ for the following equation:

$$
\left(\phi_{p}\left(x^{\prime}(t)\right)\right)^{\prime}+f\left(x^{\prime}(t)\right)+g(x(t))+\frac{h(t)}{1-x(t)}=e_{k}(t)
$$


where $e_{k}: \mathbb{R} \longrightarrow \mathbb{R}$ is a $2 k T$-periodic extension such that

$$
e_{k}(t)= \begin{cases}e(t), & t \in\left[-k T, k T-\varepsilon_{0}\right), \\ e\left(k T-\varepsilon_{0}\right)+\frac{h(-k T)-h\left(k T-\varepsilon_{0}\right)}{\varepsilon_{0}}\left(t-k T+\varepsilon_{0}\right), & t \in\left[k T-\varepsilon_{0}, k T\right],\end{cases}
$$

here $\varepsilon_{0} \in(0, T / 2)$ is a constant.

In the present paper, we list the following assumptions:

$\left(\mathrm{H}_{1}\right) . f: \mathbb{R} \longrightarrow \mathbb{R}$ is a continuous bounded nonnegative function

$\left(\mathrm{H}_{2}\right) . g: \mathbb{R} \longrightarrow \mathbb{R}$ is strictly monotone increasing and there are positive constants $\sigma$ and $n$ such that

$$
x g(x) \geq \sigma|x|^{n+1} \text { for } x \in \mathbb{R}
$$

$\left(\mathrm{H}_{3}\right)$.

$$
\sup _{t \in \mathbb{R}}|e(t)|=\rho_{1}<+\infty, \int_{\mathbb{R}}|e(t)|^{\frac{n+1}{n}} d t=\rho_{2}<+\infty, n>0
$$

\section{Main Results}

Let $y(t)=1-x(t)$, then (11) is changed into the following form:

$$
\left(\phi_{p}\left(-y^{\prime}(t)\right)\right)^{\prime}+f\left(-y^{\prime}(t)\right)+g(1-y(t))+\frac{h(t)}{y(t)}=e_{k}(t) .
$$

Obviously, the existence of $2 k T$-periodic solutions to (2) is a transfer to the existence of $2 k T$-periodic solutions to (15). For (15), consider the corresponding parameter equation:

$$
\begin{aligned}
& \left(\phi_{p}\left(-y^{\prime}(t)\right)\right)^{\prime}+\lambda f\left(-y^{\prime}(t)\right) \\
& \quad+\lambda g(1-y(t))+\lambda \frac{h(t)}{y(t)}=\lambda e_{k}(t), \lambda \in(0,1] .
\end{aligned}
$$

Here, we give the main results of the present paper in the following theorem.

Theorem 4. Assume that the assumptions $\left(\mathrm{H}_{1}\right)-\left(\mathrm{H}_{3}\right)$ hold. Then, Equation (2) has at least one positive $T$-periodic solution, if $\rho_{1}>f(0), h_{l} / \rho_{1}-f(0)<1, h_{l}=\min _{t \in \mathbb{R}} h(t)$.

Proof. Let

$$
\begin{aligned}
\Omega= & \left\{x:\left(\phi_{p}\left(-y^{\prime}(t)\right)\right)^{\prime}+\lambda f\left(-y^{\prime}(t)\right)+\lambda g(1-y(t))+\lambda \frac{h(t)}{y(t)}\right. \\
& =\lambda e_{k}(t), \lambda \in(0,1], x(t+2 k T) \\
& =x(t), x(t)>0, t \in[-k T, k T], k \in N\}
\end{aligned}
$$

with the norm

$$
|\varphi|_{\infty}=\max _{t \in[-k T, k T]}\left\{|\varphi|_{0},\left|\varphi^{\prime}\right|_{0}\right\}
$$

Let $u \in \Omega$, then $u$ satisfies

$$
\left(\phi_{p}\left(-u^{\prime}(t)\right)\right)^{\prime}+\lambda f\left(-u^{\prime}(t)\right)+\lambda g(1-u(t))+\lambda \frac{h(t)}{u(t)}=\lambda e_{k}(t) .
$$

There exist $t_{1}, t_{2} \in[-k T, k T]$ such that

$$
u\left(t_{1}\right)=\max _{t \in[-k T, k T]} u(t), u\left(t_{2}\right)=\min _{t \in[-k T, k T]} u(t) .
$$

This implies that

$$
u^{\prime}\left(t_{1}\right)=u^{\prime}\left(t_{2}\right)=0
$$

By (19), we have

$$
\begin{aligned}
& f(0)+g\left(1-u\left(t_{1}\right)\right)+\frac{h\left(t_{1}\right)}{u\left(t_{1}\right)}=e_{k}\left(t_{1}\right), \\
& g\left(1-u\left(t_{1}\right)\right) \geq-\left|e_{k}\right|_{0}-f(0)=-\rho_{1}-f(0) .
\end{aligned}
$$

In view of monotonicity of $g$, it follows by (22) that

$$
u\left(t_{1}\right)<1-g^{-1}\left(-\rho_{1}-f(0)\right):=A_{1} .
$$

On the other hand,

$$
f(0)+g\left(1-u\left(t_{2}\right)\right)+\frac{h\left(t_{2}\right)}{u\left(t_{2}\right)}=e_{k}\left(t_{2}\right)
$$

We claim that

$$
u\left(t_{2}\right)>\frac{h_{l}}{\rho_{1}-f(0)}:=A_{2} .
$$

In fact, if (26) is not true, then

$$
u\left(t_{2}\right) \leq \frac{h_{l}}{\rho_{1}-f(0)}<1 .
$$


By (25), we have

$g\left(1-u\left(t_{2}\right)\right) \leq \rho_{1}-f(0)-\frac{h_{l}}{u\left(t_{2}\right)}, \leq \rho_{1}-f(0)-\left(\rho_{1}-f(0)\right),=0$.

Thus, we have $u\left(t_{2}\right) \geq 1$ which is a contradiction to (27). From (24) and (26), we have

$$
A_{2}<u(t)<A_{1} \text { for all } t \in[-k T, k T] \text {. }
$$

Now, we estimate the bound of $u^{\prime}(t)$. For $i \in\{-k,-k+$ $1, \cdots, k-1\}$, there exists $t_{i} \in[i T,(i+1) T]$ such that

$$
u^{\prime}\left(t_{i}\right)=\frac{1}{T} \int_{i T}^{(i+1) T} u^{\prime}(s) d s
$$

It follows from (29) and (30) that

$\left|u^{\prime}\left(t_{i}\right)\right|=\left|\frac{1}{T} \int_{i T}^{(i+1) T} u^{\prime}(s) d s\right|,=\frac{1}{T}|u(i T)-u((i+1) T)|<\frac{2 A_{1}}{T}$.

Integrating (19) over $\left[t_{i}, t\right]$, we have

$$
\begin{aligned}
\phi_{p}\left(-u^{\prime}(t)\right)= & \phi_{p}\left(-u^{\prime}\left(t_{i}\right)\right)-\lambda \int_{t_{i}}^{t} f\left(-u^{\prime}(s)\right) d s, \\
& -\lambda \int_{t_{i}}^{t} g(1-u(s)) d s-\lambda \int_{t_{i}}^{t} \frac{h(s)}{u(s)} d s \\
& +\lambda \int_{t_{i}}^{t} e_{k}(s) d s, \\
\left|u^{\prime}(t)\right|^{p-1} \leq & \left(\frac{2 A_{1}}{T}\right)^{p-1}+\int_{i T}^{(i+1) T}\left|f\left(-u^{\prime}(s)\right)\right| d s, \\
& +\int_{i T}^{(i+1) T}|g(1-u(s))| d s+\int_{i T}^{(i+1) T}\left|\frac{h(s)}{u(s)}\right| d s \\
& +\int_{i T}^{(i+1) T}\left|e_{k}(s)\right| d s, \leq\left(\frac{2 A_{1}}{T}\right)^{p-1} \\
& +T f_{m}+T g_{A_{2}, A_{1}}+\frac{T h_{m}}{A_{2}}+T \rho_{1}:=A_{3},
\end{aligned}
$$

where $g_{A_{2}, A_{1}}=\max _{A_{2} \leq u \leq A_{1}}|g(1-u)|, f_{m}=\max _{s \in \mathbb{R}}|f(s)|$, $h_{m}=\max _{s \in \mathbb{R}}|h(s)|$. Thus,

$$
\left|u^{\prime}\right|_{0}=\max _{t \in[-k T, k T]}\left|u^{\prime}(t)\right| \leq\left(A_{3}\right)^{1 /(p-1)}
$$

Let $|u|_{\infty}=\max \left\{A_{1}, A_{2},\left(A_{3}\right)^{1 /(p-1)}\right\}+1$ for $u \in \Omega$. Then, condition (1) of Theorem 1 holds. Next, let

$$
\mathscr{F}(a)=f(0)+g(1-a)+\frac{\bar{h}}{a}-\overline{e_{k}}=0, a \in \mathbb{R} .
$$

Clearly, equation (34) has no solution on $\partial \Omega \cap \mathbb{R}$. Hence, condition (2) of Theorem 1 holds. Furthermore, by $\bar{h}>0$ and (29), we have the following inequalities:

$$
\begin{aligned}
& f(0)+g(1-u)+\frac{\bar{h}}{u}-\overline{e_{k}}>0 \text { for } u \in\left(0, A_{2}\right], \\
& f(0)+g(1-u)+\frac{\bar{h}}{u}-\overline{e_{k}}<0 \text { for } u \in\left[A_{1},+\infty\right) .
\end{aligned}
$$

Thus

$$
\left(f(0)+g\left(1-A_{2}\right)+\frac{\bar{h}}{A_{2}}-\overline{e_{k}}\right)\left(f(0)+g\left(1-A_{1}\right)+\frac{\bar{h}}{A_{1}}-\overline{e_{k}}\right)<0,
$$

and $d_{B}\left(\mathscr{F},\left(\gamma_{0}, M_{1}\right) \cap \mathbb{R}, 0\right) \neq 0$, i.e., condition (3) of Theorem 1 holds. By using Theorem 1, we see that Equation (15) exists at least one positive $2 k T$-periodic solution $u_{k}(t)$ such that

$$
A_{2} \leq u_{k}(t) \leq A_{1},\left|u_{k}^{\prime}(t)\right| \leq\left(A_{3}\right)^{1 / p-1}, k \in N
$$

Since $y(t)=1-x(t)$, there exist positive constants $B_{1}$, $B_{2}$, and $B_{3}$ such that

$$
B_{1} \leq \omega_{k}(t) \leq B_{2},\left|\omega_{k}^{\prime}(t)\right| \leq B_{3}, k \in N,
$$

where $\omega_{k}(t)$ is $2 k T$-periodic solution to (11). Thus,

$$
\left(\phi_{p}\left(\omega_{k}^{\prime}(t)\right)\right)^{\prime}+f\left(\omega_{k}^{\prime}(t)\right)+g\left(\omega_{k}(t)\right)+\frac{h(t)}{1-\omega_{k}(t)}=e_{k}(t),
$$

In view of Lemma 3 , there exist $\omega_{0} \in C^{1}(\mathbb{R}, \mathbb{R})$ and a subsequence $\left\{\omega_{k_{i}}\right\}$ of $\left\{\omega_{k}\right\}$ such that

$$
\begin{aligned}
& \max _{t \in[-j T, j T]}\left|\omega_{k_{i}}(t)-\omega_{0}(t)\right| \longrightarrow 0, \\
& \max _{t \in[-j T, j T]}\left|\omega_{k_{i}}{ }^{\prime}(t)-\omega_{0}^{\prime}(t)\right| \longrightarrow 0 \text { as } t \longrightarrow+\infty, j \in N .
\end{aligned}
$$

From (39), (40), and the standard argument, $\omega_{0}(t)$ is a solution of (11), i.e.,

$$
\begin{aligned}
& \left(\phi_{p}\left(\omega_{0}{ }^{\prime}(t)\right)\right)^{\prime}+f\left(\omega_{0}{ }^{\prime}(t)\right) \\
& \quad+g\left(\omega_{0}(t)\right)+\frac{h(t)}{1-\omega_{0}(t)}=e(t), t \in \mathbb{R} .
\end{aligned}
$$


Now, we will show

$$
\begin{aligned}
& \omega_{0}(t) \longrightarrow 0 \\
& \omega_{0}^{\prime}(t) \longrightarrow 0 \text { as }|t| \longrightarrow+\infty .
\end{aligned}
$$

Multiplying (39) by $\omega_{k}(t)$ and integrating it over $[-k$ $T, K T]$, we have

$$
\begin{aligned}
& \int_{-k T}^{k T}\left|\omega_{k}^{\prime}(t)\right|^{p}+\int_{-k T}^{k T} f\left(\omega_{k}^{\prime}(s)\right) \omega_{k}(s) d s \\
& \quad+\int_{-k T}^{k T} g\left(\omega_{k}(s)\right) \omega_{k}(s) d s+\int_{-k T}^{k T} \frac{h(s) \omega_{k}(s)}{1-\omega_{k}(s)} d s \\
& \quad=\int_{-k T}^{k T} e_{k}(s) \omega_{k}(s) d s .
\end{aligned}
$$

From (43), assumptions $\left(\mathrm{H}_{1}\right)$ and $\left(\mathrm{H}_{2}\right)$, we have

$$
\begin{aligned}
& \int_{-k T}^{k T}\left|\omega_{k^{\prime}}(t)\right|^{p}+\sigma \int_{-k T}^{k T}\left|\omega_{k}(s)\right|^{n+1} d s \\
& \quad \leq \int_{-k T}^{k T}\left|e_{k}(s) \omega_{k}(s)\right| d s \\
& \quad \leq\left(\int_{-k T}^{k T}\left|e_{k}(s)\right|^{n+1 / n} d s\right)^{n / n+1}\left(\int_{-k T}^{k T}\left|\omega_{k}(s)\right|^{n+1} d s\right)^{1 / n+1} .
\end{aligned}
$$

In view of $\left(\mathrm{H}_{3}\right)$ and $(12)$, we have

$$
\begin{aligned}
& \int_{-k T}^{k T}\left|e_{k}(s)\right|^{(n+1) / n} d s \\
& \quad=\int_{-k T}^{k T-\varepsilon_{0}}\left|e_{k}(s)\right|^{(n+1) / n} d s+\int_{k T-\varepsilon_{0}}^{k T}\left|e_{k}(s)\right|^{(n+1) / n} d s \\
& \quad \leq \int_{-k T}^{k T-\varepsilon_{0}}|e(s)|^{(n+1) / n} d s+\varepsilon_{0} \rho_{1}^{(n+1) / n} \\
& \quad \leq \int_{\mathbb{R}}|e(s)|^{(n+1) / n} d s+\varepsilon_{0} \rho_{1}^{(n+1) / n} \\
& \quad \leq \rho_{2}+\varepsilon_{0} \rho_{1}^{(n+1) / n}:=C_{1} .
\end{aligned}
$$

In view of (44) and (45), then

$$
\begin{gathered}
\sigma \int_{-k T}^{k T}\left|\omega_{k}(s)\right|^{n+1} d s \leq C_{1}^{n /(n+1)}\left(\int_{-k T}^{k T}\left|\omega_{k}(s)\right|^{n+1} d s\right)^{1 /(n+1)}, \\
\int_{-k T}^{k T}\left|\omega_{k}^{\prime}(t)\right|^{p} \leq C_{1}^{n /(n+1)}\left(\int_{-k T}^{k T}\left|\omega_{k}(s)\right|^{n+1} d s\right)^{1 /(n+1)} .
\end{gathered}
$$

It follows by (46) that

$$
\int_{-k T}^{k T}\left|\omega_{k}(s)\right|^{n+1} d s \leq C_{1} \sigma^{-(n+1) / n}, k \in N
$$

In view of (47) and (48), then

$$
\int_{-k T}^{k T}\left|\omega_{k}^{\prime}(t)\right|^{p} \leq C_{1} \sigma^{-1 / n}, k \in N
$$

From (48), (49) and standard limit analysis, we have $\int_{-\infty}^{+\infty}\left|\omega_{0}(s)\right|^{n+1} d s+\int_{-\infty}^{+\infty}\left|\omega_{0^{\prime}}(s)\right|^{p} d s \leq C_{1} \sigma^{-(n+1) / n}+C_{1} \sigma^{-1 / n}$

$$
\int_{|t|>r}\left(\left|\omega_{0}(s)\right|^{n+1}+\left|\omega_{0^{\prime}}(s)\right|^{p}\right) d s \longrightarrow 0 \text { as } r \longrightarrow+\infty
$$

which together with Lemma 2 yields that

$$
\begin{aligned}
\left|\omega_{0}(t)\right| \leq & (2 a)^{-1 /(n+1)}\left(\int_{t-a}^{t+a}\left|\omega_{0}(s)\right|^{n+1} d s\right)^{1 /(n+1)} \\
& +a(2 a)^{-1 / p}\left(\int_{t-a}^{t+a}\left|\omega_{0}^{\prime}(s)\right|^{p} d s\right)^{1 / p} \\
\leq & \max \left\{(2 a)^{-1 /(n+1)}, a(2 a)^{-1 / p}\right\} \\
& \cdot\left[\left(\int_{t-a}^{t+a}\left|\omega_{0}(s)\right|^{n+1} d s\right)^{1 /(n+1)}\right. \\
& \left.+\left(\int_{t-a}^{t+a}\left|\omega_{0}^{\prime}(s)\right|^{p} d s\right)^{1 / p}\right] \longrightarrow 0 \text { as }|t| \longrightarrow+\infty .
\end{aligned}
$$

Thus,

$$
\left|\omega_{0}(t)\right| \longrightarrow 0 \text { as }|t| \longrightarrow+\infty
$$

Next, we prove

$$
\left|\omega_{0}^{\prime}(t)\right| \longrightarrow 0 \text { as }|t| \longrightarrow+\infty .
$$

Furthermore, by (38) we have

$$
B_{1} \leq \omega_{0}(t) \leq B_{2},\left|\omega_{0}^{\prime}(t)\right| \leq B_{3}, t \in \mathbb{R}
$$

which together with (41) yields that

$$
\left|\left(\phi_{p}\left(\omega_{0}^{\prime}(t)\right)\right)^{\prime}\right| \leq f_{B_{3}}+g_{B_{1}, B_{2}}+\frac{h_{m}}{B_{1}-1}+|e|_{0}:=C_{2},
$$


where $f_{B_{3}}=\max _{|s| \leq B_{3}}|f(s)|, g_{B_{1}, B_{2}}=\max _{B_{1} \leq s \leq B_{2}}|g(s)|$. If (54) does not hold. Then, there are constant $\delta \in(0,1 / 2)$ and a sequence $\left\{t_{k}\right\}$ with $\left|t_{1}\right|<\left|t_{2}\right|<\cdots$ such that

$$
\begin{aligned}
\left|\phi_{p}\left(\omega_{0^{\prime}}\left(t_{k}\right)\right)\right| & \geq 2 \delta, k=1,2, \cdots \\
\left|\phi_{p}\left(\omega_{0}^{\prime}(t)\right)\right| & =\left|\phi_{p}\left(\omega_{0^{\prime}}\left(t_{k}\right)\right)+\int_{t_{k}}^{t}\left(\phi_{p}\left(\omega_{0^{\prime}}(s)\right)\right)^{\prime} d s\right|, \\
& \geq\left|\phi_{p}\left(\omega_{0^{\prime}}\left(t_{k}\right)\right)\right|-\int_{t_{k}}^{t}\left|\left(\phi_{p}\left(\omega_{0^{\prime}}(s)\right)\right)^{\prime}\right| d s, \\
& \geq \delta \text { for } t \in\left[t_{k}, t_{k}+\frac{\delta}{1+C_{2}}\right], \\
\int_{-\infty}^{+\infty}\left|\phi_{p}\left(\omega_{0^{\prime}}(t)\right)\right| d t & \geq \sum_{k=1}^{+\infty} \int_{t_{k}}^{t_{k}+\left(\delta /\left(1+C_{2}\right)\right)}\left|\phi_{p}\left(\omega_{0^{\prime}}(t)\right)\right| d t=+\infty,
\end{aligned}
$$

which contradicts to (50). It is easy to see that (54) holds. Thus, $\omega_{0}(t)$ is just a homoclinic solution to Eq. (2).

\section{Examples}

This section presents two examples that demonstrate the validity of our theoretical results.

Example 5. Consider the following equation:

$$
\begin{gathered}
\left(\left|u^{\prime}(t)\right|^{2} u^{\prime}(t)\right)^{\prime}+1+2 x^{\prime 2}(t)+2 x^{3}(t) \\
+\frac{(3-(1 / 2) \cos t)}{(1-x(t))}=5+\frac{1}{2} \sin ^{2} t
\end{gathered}
$$

where

$$
\begin{aligned}
& p=4, f\left(x^{\prime}(t)\right)=1+2 x^{\prime 2}(t), g(x(t))=2 x^{3}(t) \\
& h(t)=3-\frac{1}{2} \cos t, e(t)=5+\frac{1}{2} \sin ^{2} t
\end{aligned}
$$

Obviously, $\rho_{1}=5.5, f(0)=1, h_{l}=2.5, h_{l} /\left(\rho_{1}-f(0)\right)=5 /$ $7<1$. We also easily check that assumptions $\left(\mathrm{H}_{1}\right)-\left(\mathrm{H}_{3}\right)$ hold. Based on Theorem 4, Equation (58) has at least one nontrivial homoclinic solution.

Example 6. Consider the following equation:

$u^{\prime \prime}(t)+3+2 x^{\prime 2}(t)+4 x^{3}(t)+\frac{(5-(1 / 2) \sin t)}{(1-x(t))}=8+\frac{1}{2} \cos ^{2} t$

where

$$
\begin{aligned}
& p=2, f\left(x^{\prime}(t)\right)=3+2 x^{\prime 2}(t), g(x(t))=4 x^{3}(t), \\
& h(t)=5-\frac{1}{2} \sin t, e(t)=8+\frac{1}{2} \cos ^{2} t
\end{aligned}
$$

Obviously, $\rho_{1}=8.5, f(0)=3, h_{l}=4.5, h_{l} /\left(\rho_{1}-f(0)\right)=9 /$ $11<1$. We also easily check that assumptions $\left(\mathrm{H}_{1}\right)-\left(\mathrm{H}_{3}\right)$ hold. Based on Theorem 4, Equation (60) has at least one nontrivial homoclinic solution.

\section{Conclusions}

In this paper, we study a class of second-order singular equation with $p$-Laplacian. By employing some analytic techniques and continuation theorem due to Mana'sevich and Mawhin, we have presented some new sufficient criteria for the existence of homoclinic solutions for the above singular equation. These criteria possess adjustable parameters which are important in some applied fields. Finally, two examples are given to demonstrate the effectiveness of the obtained theoretical results. However, there exist many problems for further study such as heteroclinic orbits of second-order singular equations.

\section{Data Availability}

No data were used to support this study.

\section{Conflicts of Interest}

The authors declare that they have no competing interests.

\section{Authors' Contributions}

All authors contributed equally to the writing of this paper. All authors read and approved the final manuscript.

\section{Acknowledgments}

The work is supported by the Natural Science Foundation of Jiangsu High Education Institutions of China (Grant No. 17KJB110001).

\section{References}

[1] P. H. Rabinowitz, "Chapter 14 Variational methods for Hamiltonian systems," in Handbook of Dynamical Systems, vol. 1, pp. 1091-1127, Elsevier, Amsterdam, 2002.

[2] P. C. Carrião and O. H. Miyagaki, "Existence of Homoclinic Solutions for a Class of Time-Dependent Hamiltonian Systems," Journal of Mathematical Analysis and Applications, vol. 230, no. 1, pp. 157-172, 1999.

[3] M. Izydorek and J. Janczewska, "Homoclinic solutions for a class of the second order Hamiltonian systems," Journal of Differential Equations, vol. 219, no. 2, pp. 375-389, 2005.

[4] S. P. Lu, "Existence of homoclinic solutions for a class of neutral functional differential equations," Acta Mathematica Sinica, English Series, vol. 28, no. 6, pp. 1261-1274, 2012.

[5] Y. Ding and Q. Guo, "Homoclinic solutions for an anomalous diffusion system," Journal of Mathematical Analysis and Applications, vol. 466, no. 1, pp. 860-879, 2018.

[6] C. Li, "Remarks on homoclinic solutions for semilinear fourth-order ordinary differential equations without periodicity," Applied Mathematics-A Journal of Chinese Universities, vol. 24, no. 1, pp. 49-55, 2009. 
[7] B. Du, "Anti-periodic solutions problem for inertial competitive neutral-type neural networks via Wirtinger inequality," Journal of Inequalities and Applications, vol. 2019, no. 1, 2019.

[8] J. Sun, H. Chen, and J. J. Nieto, "Homoclinic solutions for a class of subquadratic second-order hamiltonian systems," Journal of Mathematical Analysis and Applications, vol. 373, no. 1, pp. 20-29, 2011.

[9] T. Zhou, B. Du, and H. Du, "Positive periodic solution for indefinite singular Liénard equation with p-Laplacian," Advances in Difference Equations, vol. 2019, no. 1, Article ID $158,2019$.

[10] W. Omana and M. Willem, "Homoclinic orbits for a class of hamiltonian systems," Integral Equation, vol. 5, no. 5, pp. 1115-1120, 1992

[11] D. Bonheure and P. J. Torres, "Bounded and homoclinic-like solutions of a second-order singular differential equation," Bulletin of the London Mathematical Society, vol. 44, no. 1, pp. 47-54, 2012.

[12] T. Bartsch and A. Szulkin, "Chapter 2 Hamiltonian Systems: Periodic and Homoclinic Solutions by Variational Methods," Handbook of Differential Equations: Ordinary Differential Equations, pp. 77-146, Elsevier, 2005.

[13] D. Bonheure and L. Sanchez, "Heteroclinic orbits for some classes of second and fourth order differential equations," in Handbook of differential equations: ordinary differential equations, pp. 103-202, 2006.

[14] D. G. Costa and H. Tehrani, "On a class of singular secondorder Hamiltonian systems with infinitely many homoclinic solutions," Journal of Mathematical Analysis and Applications, vol. 412, no. 1, pp. 200-211, 2014.

[15] U. Bessi, "Multiple homoclinic orbits for autonomous, singular potentials," Proceedings of the Royal Society of Edinburgh: Section A Mathematics, vol. 124, no. 4, pp. 785-802, 1994.

[16] M. J. Borges, "Heteroclinic and homoclinic solutions for a singular Hamiltonian system," European Journal of Applied Mathematics, vol. 17, no. 1, pp. 1-802, 2006.

[17] R. Manásevich and J. Mawhin, "Periodic Solutions for Nonlinear Systems with $p$-Laplacian-Like Operators," Journal of Differential Equations, vol. 145, no. 2, pp. 367-393, 1998.

[18] R. Gaines and J. Mawhin, Coincidence Degree and Nonlinear Differential Equations, Springer, Berlin, 1977.

[19] X. H. Tang and L. Xiao, "Homoclinic solutions for ordinary p-Laplacian systems with a coercive potential," Nonlinear Analysis: Theory, Methods \& Applications, vol. 71, no. 3-4, pp. 1124-1132, 2009. 